\title{
A METAPHOR FOR ADIABATIC EVOLUTION TO SYMMETRY
}

\author{
R. J. A. G. HUVENEERS ${ }^{\dagger}+$ AND F. VERHULST ${ }^{\dagger}$
}

\begin{abstract}
In this paper we study a Hamiltonian system with a spatially asymmetric potential. We are interested in the effects on the dynamics when the potential becomes symmetric slowly in time. We focus on a highly simplified non-trivial model problem (a metaphor) to be able to pursue explicit calculations as far as possible. Using the techniques of averaging and adiabatic invariants, we are able to study all bounded solutions, which reveals significant asymmetric dynamics even when the asymmetric contributions to the potential have become negligibly small.
\end{abstract}

Key words. adiabatic, invariant, averaging, symmetry

AMS subject classifications. 34C15, 34C 29, 34C35, 34E05, 34E10, 34E15

1. Introduction. Many physical objects exhibit some form of symmetry. Most galaxies for instance, have axes or planes of symmetry. The motivation for this study is that a symmetric equilibrium configuration generally is the outcome of the evolution from an asymmetric state. We would like to trace the effect of the asymmetries.

A problem is that studies of the evolution of actual physical systems are difficult and so relatively rare. We propose therefore to ignore, at least for the time being, the actual physical mechanisms and to consider systems described by a Hamiltonian of the form

$$
\mathcal{H}(p, q, \epsilon t)=\mathcal{H}_{s}(p, q)+a(\epsilon t) \mathcal{H}_{a}(p, q)
$$

where $\mathcal{H}_{s}$ is the part of the Hamiltonian which is symmetric in some sense; $\mathcal{H}_{a}$ is the asymmetric part which is slowly vanishing as we put

$$
a(0)=1, \lim _{t \rightarrow \infty} a(\epsilon t)=0,0<\epsilon \ll 1
$$

To study the dynamics induced by the Hamiltonian $\mathcal{H}(p, q, \epsilon t)$ is still a formidable problem. So we simplify as much as possible to obtain

$$
\left\{\begin{array}{l}
\dot{x}_{1}=x_{2} \\
\dot{x}_{2}=-x_{1}+a(\epsilon t) x_{1}^{2}
\end{array}\right.
$$

which is derived from the one degree of freedom Hamiltonian

$$
\mathcal{H}(p, q, \epsilon t)=\frac{1}{2}\left(p^{2}+q^{2}\right)-\frac{1}{3} a(\epsilon t) q^{3}
$$

identifying $p=x_{2}, q=x_{1}$. We shall associate with system (1.3) the "unperturbed" system which arises for $\epsilon=0$

$$
\left\{\begin{array}{l}
\dot{x}_{1}=x_{2} \\
\dot{x}_{2}=-x_{1}+x_{1}^{2}
\end{array}\right.
$$




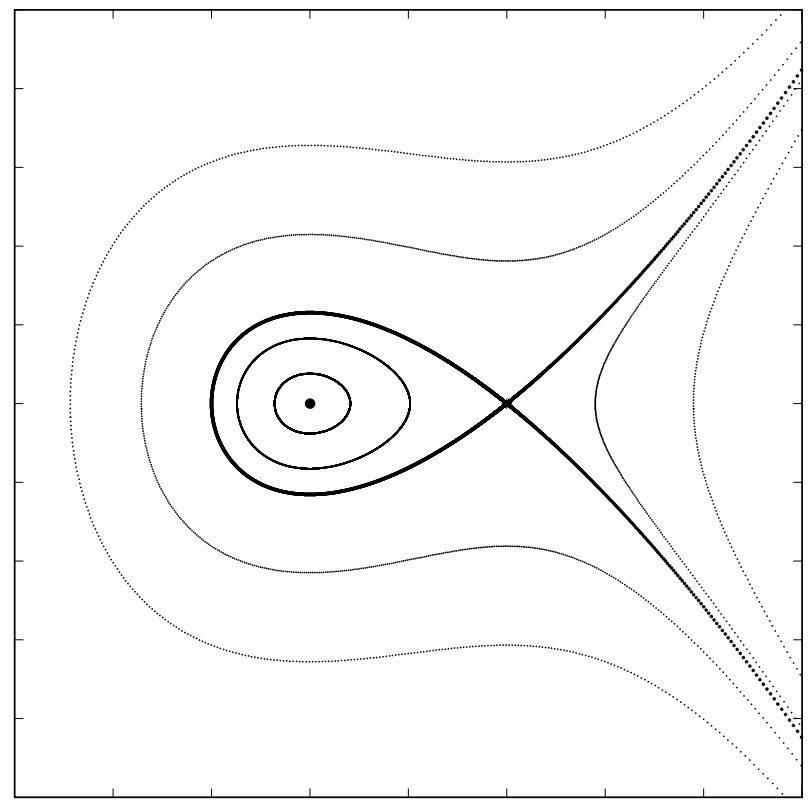

FIG. 1.1. The dynamics of the unperturbed system (1.5)

We note that in the autonomous system (1.5) there are basically two regions (figure 1.1): within the homoclinic solution the orbits are bounded, outside the homoclinic solution the orbits diverge to infinity (with the exception of the stable manifold and the saddle point itself). In system (1.3) we have no fixed saddle point, still it turns out that we have two separate regions of initial values in which the orbits are bounded or diverge to infinity.

Since the dynamics of systems (1.3) and (1.5) are the same on an $O(1)$ timescale, it is instructive (though slightly wrong) to view system (1.3) as having a saddle point moving slowly towards infinity and having a slowly expanding homoclinic orbit. Within this picture, an orbit can remain bounded in two ways, either by starting inside the homoclinic orbit, or by getting "captured" by the slowly expanding homoclinic orbit, which can only happen if the orbit starts sufficiently close to the stable manifold of the saddle point. To make these statements mathematically correct, one should use the concept of normally hyperbolic motion.

Using a special transformation we shall discuss the boundaries of these regions in section 2. A special case, $a(\epsilon t)=\exp (-\epsilon t)$ can be studied easily and help us to understand the general case.

In section 3 we perform averaging in the so-called stable region where bounded solutions are found. This involves the use of elliptic and hypergeometric functions, rather hard analysis, where we are supported by Mathematica 2.2 running under SunOS 4.1.3_U1.

After determining the validity of the averaged equation we establish the existence of an adiabatic invariant in the stable region, valid for all time. Even more remarkable is that explicit calculations of this invariant show that the evolution of phase points

\footnotetext{
$\dagger$ Department of Mathematics, University of Utrecht, P.O. Box 80.010, 3508 TA Utrecht, The Netherlands

$\ddagger$ Supported by NWO-grant nr. 611-306-537
} 
will show significant traces of its asymmetrical past for all time.

In section 4 we need subtle reasoning to discuss what is going on in the boundary layer near the boundary of the stable domain.

The analysis in this paper is based on averaging methods but, because of its direct relation to dissipative mechanics (section 2), it clearly profits from the results by Haberman and Ho [9, 10] and Bourland and Haberman [2]. Rand [16, 17] used a different approach (Jacobian elliptic functions) to study a similar class of dynamical systems.

Some aspects of these problems have been analyzed previously. Equation (1.3) is equivalent to the slowly varying elliptic functions first analyzed by Kuzmak [13] and reported in the first edition of the perturbation methods text by Cole [5] and later in the second edition by Kevorkian and Cole [12]. In Haberman [7] it is briefly remarked that Kuzmak's results show that the solution can evolve through a separatrix when the parameters are slowly varying (before the separatrix disappears). Distortions of homoclinic orbits due to perturbations are well-known when the perturbation is dissipative, and the change in energy is now called the Melnikov [14] integral, though the results are much older. In this paper, the stable and unstable manifold break apart due to slowly varying coefficients, which was perhaps first described by Haberman [8] and Robinson [18]. An early description of crossing a separatrix for slowly varying Hamiltonian systems was given by Tennyson, Cary and Escande [20] who used earlier work by Timofeev [21]. We also mention Neishtadt [15] and Henrard [11] who added many applications and some theoretical aspects. Bourland and Haberman $[2,4]$ analyzed the crossing of a separatrix due to a dissipative perturbation and determined an analytical formula for the boundaries of the basins of attraction, the stable manifold of the saddle point, using multiple scales and averaging (with Kath). Bourland and Haberman [3] generalized this to include both slow variation and a dissipative perturbation.

We finally note that in the context of galactic dynamics, some rather different examples based on classical results of the theory of adiabatic invariants were given by Binney and Tremaine [1].

2. The boundary of the stable part of phase space. As we explained in the introduction, the phase space of system (1.3) can be separated into two parts. Since we are dealing with a time-dependent system, we must specify the time for which a particular separation holds. We use the following definition:

The stable part of phase space consists of the points $\left(x_{1}, x_{2}\right)$, for which the orbit $\gamma\left(x_{1}, x_{2}, 0\right)$ starting in $\left(x_{1}, x_{2}\right)$ at $t=0$ remains bounded for $t$ going to infinity. All other points define the unstable part of phase space.

Clearly, a point $\left(x_{1}, x_{2}\right)$ can only be contained in the stable region if it lies within an $O(\epsilon)$ neighbourhood of the area bounded by the homoclinic orbit of system (1.5). If this is not the case, $\gamma\left(x_{1}, x_{2}, 0\right)$ will reach the upper branch of the unstable manifold of the saddle point of system (1.5) in a finite time and clear off to infinity. We must not overlook the orbits starting close to the lower branch of the stable manifold of the saddle point of system (1.5), which can reach the just described $O(\epsilon)$ neighbourhood too. It will turn out that although this region may look small, it produces the major part of the stable region.

These considerations help us locating the boundary of the stable region approximately. The location of the boundary of the stable region separates the part of phase space in which all orbits diverge to infinity $\left(\left(x_{1}, x_{2}\right) \rightarrow(+\infty,+\infty)\right)$ from the part of phase space in which the orbits tend to circle around the origin for $t$ going to infinity, 
so if we expect to see effects of the vanishing of the asymmetric potential somewhere, it is just within this boundary.

The key step in analyzing system (1.3) is performing the transformation

$$
y_{1}=a(\epsilon t) x_{1}
$$

The idea behind this transformation is to try to fix the normally hyperbolic motion of system (1.3). Since we want to study system (1.3) for all time, this time-dependent rescaling of the coordinates enables us to study a bounded domain, which simplifies the calculations considerably. This transformation has also disadvantages, the biggest of which is probably the loss of the Hamiltonian structure, as discussed below.

Demanding that $\dot{y}_{1}=y_{2}$, we arrive at the system

$$
\left\{\begin{array}{l}
\dot{y}_{1}=y_{2} \\
\dot{y}_{2}=-y_{1}+y_{1}^{2}+2 \epsilon \frac{a^{\prime}(\epsilon t)}{a(\epsilon t)} y_{2}+\epsilon^{2}\left(\frac{a^{\prime \prime}(\epsilon t)}{a(\epsilon t)}-2 \frac{a^{\prime}(\epsilon t)^{2}}{a(\epsilon t)^{2}}\right) y_{1}
\end{array}\right.
$$

where $a^{\prime}(\epsilon t)$ stands for $\left.\frac{\mathrm{d} a(\xi)}{\mathrm{d} \xi}\right|_{\xi=\epsilon t}$ and similarly for $a^{\prime \prime}(\epsilon t)$.

By transformation (2.1) the slow time-dependence has moved to $O(\epsilon)$ terms; still, system (2.2) looks more complicated than system (1.3). However, we will be able to neglect the $O\left(\epsilon^{2}\right)$ term in most of our calculations. We should also note that system (2.2) is not Hamiltonian anymore, since we have applied a non-canonical transformation. Indeed the $O(\epsilon)$ term is a friction term, causing the origin $\left(y_{1}, y_{2}\right)=(0,0)$ to become an attracting focus instead of a center.

To guarantee that capturing occurs in the neighbourhood of the homoclinic orbit, we demand that the damping-coefficient $\left(\frac{a^{\prime}}{a}\right)$ be negative for all time. This ensures that captured orbits will not be able to escape the homoclinic orbit. Since $a(0)=1$ this condition is equivalent to demanding that a be strictly monotonically decreasing. Allowing the damping-coefficient to become positive would destroy the capturing effect for certain or even all orbits.

In the analysis of system (2.2) we start with a special choice of $a(\epsilon t)$.

2.1. The special case $a(\epsilon t)=e^{-\epsilon t}$. We will first calculate the location of the boundary of the stable region for the special, but physically important case

$$
a(\epsilon t)=e^{-\epsilon t}
$$

We will show later that the general case does not differ much from this special case. With the choice $(2.3)$ for $a(\epsilon t)$, system $(2.2)$ reduces to

$$
\left\{\begin{array}{l}
\dot{y}_{1}=y_{2} \\
\dot{y}_{2}=-y_{1}+y_{1}^{2}-2 \epsilon y_{2}-\epsilon^{2} y_{1}
\end{array}\right.
$$

It is remarkable that for this special yet interesting choice of $a(\epsilon t)$, our system becomes autonomous, which reduces the calculations because the dependence on the initial time has vanished into the transformation (2.1).

We also note that we have succeeded in fixing the saddle point: The saddle point of system $(2.4)$ is located in $\left(1+\epsilon^{2}, 0\right)$. 


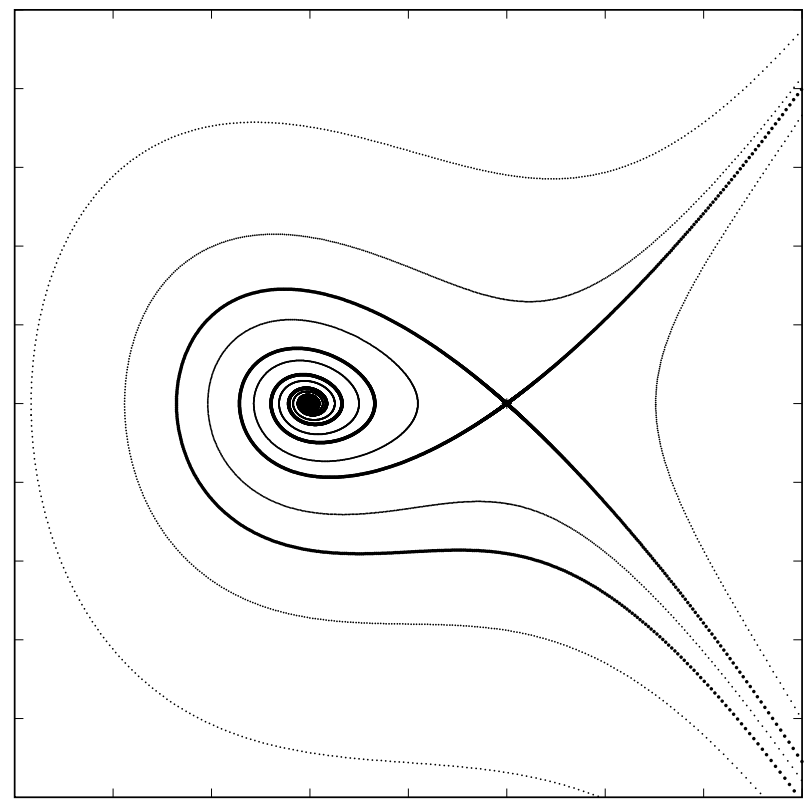

FIG. 2.1. The stable and unstable manifold of system (2.5) with $\epsilon=0.1$

The saddle point not being located in $(1,0)$ as we intended would introduce a lot of extra small terms in our calculations. To avoid these we map the saddle point onto $(1,0)$ by substituting $y_{i} \rightarrow\left(1+\epsilon^{2}\right) y_{i}, i=1,2$, to obtain

$$
\left\{\begin{array}{l}
\dot{y}_{1}=y_{2} \\
\dot{y}_{2}=-\left(1+\epsilon^{2}\right)\left(y_{1}-y_{1}^{2}\right)-2 \epsilon y_{2}
\end{array}\right.
$$

So we have reduced the calculation of the boundary of the stable region of system (1.3) to the calculation of the (time-independent) region of attraction of system (2.5).

It is easily seen (figure 2.1) that the region of attraction of system (2.5) is bounded by the stable manifold of the saddle point.

It is well known that generally the stable manifold of a perturbed system (with parameter $\epsilon$ ) lies in an $O(\epsilon)$ neighbourhood of the stable manifold of the unperturbed system (with $\epsilon=0$ ). The unperturbed system

$$
\left\{\begin{array}{l}
\dot{y}_{1}=y_{2} \\
\dot{y}_{2}=-y_{1}+y_{1}^{2}
\end{array}\right.
$$

is simple and totally understood. It has a first integral $E(\epsilon=0)$ where

$$
E(\epsilon=0)=\frac{1}{2} y_{2}^{2}+\frac{1}{2} y_{1}^{2}-\frac{1}{3} y_{1}^{3}
$$

and the unstable manifold coincides with the homoclinic orbit $E(\epsilon=0)=\frac{1}{6}$.

Using $E(\epsilon=0)$ in our calculations for the perturbed system would introduce some higher order terms. Instead, we extend the definition of $E$ with suitable $O\left(\epsilon^{2}\right)$ terms which cancel these terms. Again, this is only for calculational convenience. 


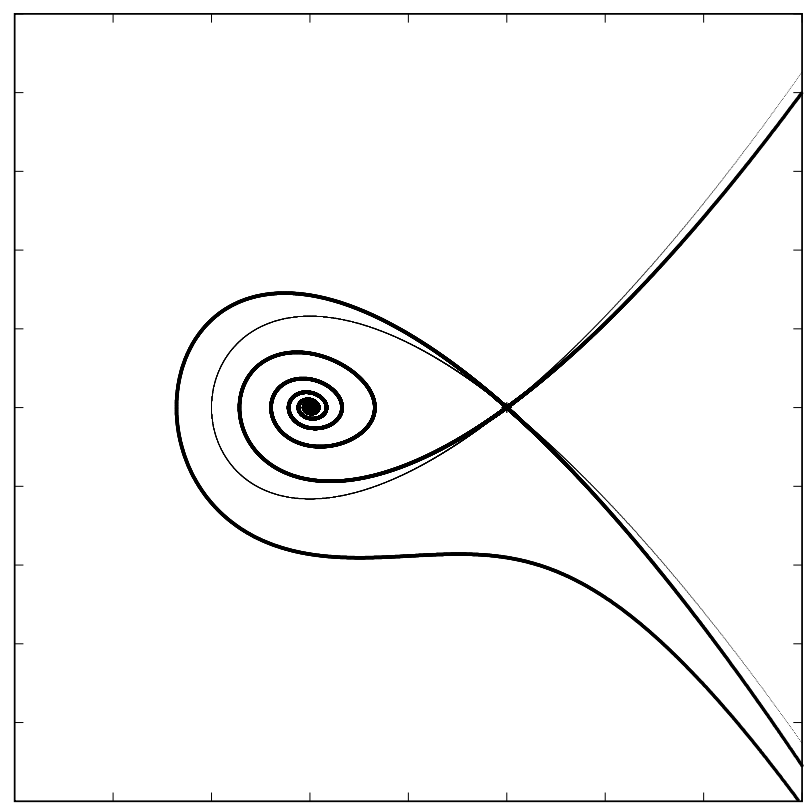

FIG. 2.2. The homoclinic orbit (represented by the thin line) of system (2.6) added to figure 2.1

$$
E=\frac{1}{2} y_{2}^{2}+\frac{1}{2}\left(1+\epsilon^{2}\right) y_{1}^{2}-\frac{1}{3}\left(1+\epsilon^{2}\right) y_{1}^{3}
$$

It is instructive to combine figure 2.1 with the homoclinic orbit of the unperturbed system (2.6), which produces figure 2.2 .

We will now approximate the location of the stable manifold of the saddle point of system (2.5) by calculating the variation of $E$ along the stable manifold. Since this variation is an $O(\epsilon)$ effect, we may use the unperturbed stable manifold in this calculation, which involves elliptic functions. From this variation of $E$ along the stable manifold, we can deduce the location of the perturbed stable manifold.

If we follow the flow along the stable manifold from a point $\left(y_{10}, y_{20}\right)$ to a point $\left(y_{11}, y_{21}\right)$ we get:

$$
\frac{\mathrm{d} E}{\mathrm{~d} t}=-2 \epsilon y_{2}^{2} \Rightarrow \Delta E=\int-2 \epsilon y_{2}^{2} d t=-2 \epsilon \int_{y_{10}}^{y_{11}} y_{2} d y_{1}
$$

The integral appearing in this expression has to be calculated with $O(\epsilon)$ precision, which allows us to substitute the explicitly known orbits of the unperturbed system (2.6). These orbits $\left(y_{2}\left(y_{1}\right)\right)$ are readily obtained from the definition of the first integral $(2.7)$.

To calculate the variation of $E$ along the upper branch of the stable manifold, we take $\left(y_{11}, y_{21}\right)=(1,0)$ and get, after some analysis as indicated above:

$(2.10) E\left(y_{1}\right)=\frac{1}{6}\left(1+\epsilon^{2}\right)+2 \epsilon\left(\frac{3}{5}+\frac{\sqrt{3}\left(y_{1}-2\right)\left(2 y_{1}+1\right)^{(3 / 2)}}{15}\right)+O\left(\epsilon^{2}\left(y_{1}-1\right)^{2}\right)$ 
which is valid for $-\frac{1}{2} \leq y_{1} \leq 1$ and $y_{2}>0$.

For $y_{1}<-\frac{1}{2}$ we have $\dot{E}=\bar{O}\left(\epsilon^{2}\right)$ and therefore we get:

$$
E\left(y_{1}\right)=\frac{1}{6}+\frac{6}{5} \epsilon+O\left(\epsilon^{2}\right)
$$

which is valid for $y_{1}<-\frac{1}{2}$

Taking $\left(y_{11}, y_{21}\right)=\left(-\frac{1}{2}, y_{21}\right)$ we get:

$$
E\left(y_{1}\right)=\frac{1}{6}+2 \epsilon\left(\frac{3}{5}-\frac{\sqrt{3}\left(y_{1}-2\right)\left(2 y_{1}+1\right)^{(3 / 2)}}{15}\right)+O\left(\epsilon^{2} \log \epsilon\right)
$$

which is valid for $-\frac{1}{2} \leq y_{1} \leq 1$ and $y_{2}<0$. The special form of the error term arises from the fact that the homoclinic orbit is only an $O(\sqrt{\epsilon})$ approximation of the stable manifold for $y_{1}$ close to 1 and negative $y_{2}$ (just under the saddle point). This follows from the analysis in Haberman and Ho [9].

Taking $\left(y_{11}, y_{21}\right)=\left(1, y_{21}\right)$ we get

$$
E\left(y_{1}\right)=\frac{1}{6}+2 \epsilon\left(\frac{9}{5}+\frac{\sqrt{3}\left(y_{1}-2\right)\left(2 y_{1}+1\right)^{(3 / 2)}}{15}\right)+O\left(\epsilon^{2} \log \epsilon\right)
$$

which is valid for $y_{1}>1$ and $y_{2}<0$.

To calculate the variation of $E$ along the lower branch of the stable manifold, we take $\left(y_{11}, y_{21}\right)=(1,0)$ and making use of the expressions for the explicitly known lower branch of the stable manifold of the unperturbed system (2.6) we find

$(2.14) E\left(y_{1}\right)=\frac{1}{6}\left(1+\epsilon^{2}\right)+2 \epsilon\left(\frac{3}{5}+\frac{\sqrt{3}\left(y_{1}-2\right)\left(2 y_{1}+1\right)^{(3 / 2)}}{15}\right)+O\left(\epsilon^{2}\left(y_{1}-1\right)^{2}\right)$

which is valid for $y_{1}>1$ and $y_{2}<0$.

So, we have now calculated the variation of $E$ all over the stable manifold of the saddle point of system (2.5). What is left to do is to deduce the location of the stable manifold itself from this variation, which is not very hard.

Given a value of $y_{1}$, one first calculates the corresponding value of $E$ using the appropriate formula given above. Using the definition of $E$ (2.8), one calculates the corresponding value of $y_{2}$. This amounts to solving a third order polynomial, which can even be done explicitly.

In particular one can compute the intersection of the stable manifold with the $y_{1}$-axis, which occurs (approximately) in $\left(-\frac{1}{2}-\frac{8}{5} \epsilon, 0\right)$.

2.2. The boundary of the stable region for arbitrary $a(\epsilon t)$. We now return to the discussion of the general system (2.2). It turns out that the analysis is essentially the same as for the special case $a(\epsilon t)=e^{-\epsilon t}$

We claim that the behaviour of system (2.2) is (with a certain error) described by the system

$$
\left\{\begin{array}{l}
\dot{y}_{1}=y_{2} \\
\dot{y}_{2}=-y_{1}+y_{1}^{2}+2 \epsilon \frac{a^{\prime}(0)}{a(0)} y_{2}
\end{array}\right.
$$




\begin{tabular}{|l|l|l|}
\hline$\epsilon$ & error & $\frac{\text { error }}{\epsilon^{2} \log \epsilon}$ \\
\hline 0.1 & 0.5951 & 25.8 \\
0.05 & 0.1447 & 19.3 \\
0.01 & 0.006362 & 13.8 \\
0.005 & 0.001695 & 12.8 \\
0.001 & 0.00007877 & 11.4 \\
0.0005 & 0.00002093 & 11.0 \\
0.0001 & 0.0000009529 & 10.4 \\
\hline
\end{tabular}

TABLE 2.1

The column headed by "error" gives the difference between the asymptotic and a very accurate numerical location of the boundary between the stable and unstable part of phase space. For an asymptotically sharp estimate, here $O\left(\epsilon^{2} \log \epsilon\right)$, we expect the ratio of this error and $\epsilon^{2} \log \epsilon$ to tend to a positive constant as $\epsilon \rightarrow 0$ (third column).

as far as the location of the boundary of the stable region is concerned.

The idea behind this statement is that if an orbit of system (2.2) starts at a distance $\delta$ inside the unstable region, it will reach the upper branch of the unstable manifold after an interval of time $O(\log \delta)$, because it has to pass the saddle point at a distance $O(\delta)$ (sometimes twice).

Using Gronwall's inequality, it is easy to show that the orbit of system (2.15) starting at the same initial point, will diverge at most $O\left(\frac{\epsilon^{2} \log \delta}{\delta}\right)$ from the exact orbit. Since we know the boundary of the stable region with precision $O\left(\epsilon^{2} \log \epsilon\right)$, we must take $\delta$ to be larger than $O\left(\epsilon^{2} \log \epsilon\right)$ for our calculations to make sense. Consequently, the orbit of system (2.15) will diverge at most $o(1)$ from the exact orbit and will thus diverge to infinity too.

Thus, a starting point $\left(y_{1}, y_{2}\right)$ lying more than $O\left(\epsilon^{2} \log \epsilon\right)$ inside the unstable region produces an orbit diverging to infinity both in system (2.2) and in system $(2.15)$.

We can apply the same argument to the stable region, which proves that the boundary of the stable region of system (2.2) coincides with the boundary of the stable region of system (2.15) up to $O\left(\epsilon^{2} \log \epsilon\right)$.

So we have the important conclusion that, to calculate the boundary of the stable region of system (2.2), we can use the formulas derived for the special case $a(\epsilon t)=e^{-\epsilon t}$ with $\epsilon$ replaced by $-\frac{a^{\prime}(0)}{a(0)} \epsilon$.

To derive the formulas for the special case $a(\epsilon t)=e^{-\epsilon t}$, we have integrated over infinite time intervals. This is not in conflict with freezing the damping coefficient at $t=0$, since the leading order contribution is produced on a $\log \epsilon$ timescale.

2.3. Comparison between asymptotic and numerical results. To illustrate the obtained asymptotic expressions, we have computed the boundary between the stable and unstable part of phase space both numerically and by using these expressions. For both cases, we computed the value of the unperturbed energy at the boundary just below the saddle-point. Table 2.1 gives the difference between these values for various values of $\epsilon$. From the table it is clear that our error-estimate is sharp.

For these particular calculations, we took $a(\epsilon t)=\frac{1}{(1+\epsilon t)^{2}}$.

3. Averaging inside the stable region for arbitrary $a(\epsilon t)$. Knowing the location of the boundary of the stable region we proceed to study the stable region 
itself (the unstable region is clearly not very interesting). We have to do this study in two parts in which we consider the interesting dynamics which takes place close to (we will make this more precise) the boundary of the stable region (i.e. in the boundary layer) and in the inner domain. At a safe distance from the boundary layer, system (2.2) will behave more and more like a harmonic oscillator. The natural way to approach such a problem is to apply the theory of averaging.

3.1. Averaging in the inner domain. Averaging in the vicinity of the origin is a simple exercise involving averaging over harmonic functions. This is not what we have in mind; we shall average over a part of the inner domain as large as possible. This involves averaging over elliptic functions.

3.1.1. Calculation of the averaged equation. To perform averaging, we need one or more quantities with a small $(O(\epsilon))$ time derivative, i.e. which depend slowly on time. A natural candidate for this quantity is the exact integral (2.7) of the unperturbed system, for which we have

$$
\frac{\mathrm{d} E}{\mathrm{~d} t}=2 \epsilon \frac{a^{\prime}(\epsilon t)}{a(\epsilon t)} y_{2}^{2}+\epsilon^{2}\left(1-y_{1}+\left(\frac{a^{\prime \prime}(\epsilon t)}{a(\epsilon t)}-2 \frac{a^{\prime}(\epsilon t)^{2}}{a(\epsilon t)^{2}}\right)\right) y_{1} y_{2}
$$

To be able to average this equation, we have to put restrictions on $a(\epsilon t)$ :

$$
\begin{aligned}
\frac{a^{\prime}(\xi)}{a(\xi)} & \text { is bounded for all positive } \xi \\
\frac{a^{\prime \prime}(\xi)}{a(\xi)} & \text { is bounded for all positive } \xi
\end{aligned}
$$

Most decaying functions of interest satisfy these restrictions. Functions decaying extremely rapidly, such as $a(\xi)=\exp (-\exp (\xi))$, do not satisfy these restrictions. But since $a(\xi)$ decays very rapidly, we can safely put $a(\xi)$ equal to zero for all $\xi$ bigger than some $\xi_{0}$ for which $a\left(\xi_{0}\right) \ll 1$, without affecting the dynamics. Other examples of functions which do not satisfy (3.2) are functions which vanish in a finite time like $a(\xi)=1-\xi$. Again we can restrict the time span such that this poses no problem.

To average equation (3.1), we consider $\tau=\epsilon t$ as an independent variable and add the equation

$$
\dot{\tau}=\epsilon
$$

Since we only have to average the $O(\epsilon)$ part of equation (3.1), we have to average $y_{2}^{2}$ along a periodic orbit of the unperturbed system (2.6). This amounts to calculating the integral of $y_{2}^{2}$ along the periodic orbit and involves the period of the periodic orbit. This is in the spirit of averaging as for instance presented in Sanders en Verhulst [19].

To calculate $\int y_{2}^{2} d t$, we make use of $\dot{y}_{1}=y_{2}$, which reduces the calculation to the action $\int y_{2} d y_{1}$. The functional dependence of $y_{2}$ on $y_{1}$ for the unperturbed system can be retrieved from the exact integral (2.7) and is the square root of a third order polynomial.

Using this, we find that we also need this standard integral

$$
\int_{a}^{b} \sqrt{(x-a)(b-x)(c-x)}=\frac{1}{24} \sqrt{6} \pi(b-a)^{2} \sqrt{c-a_{2}} F_{1}\left(-\frac{1}{2}, \frac{3}{2}, 3, \frac{b-a}{c-a}\right)
$$


with ${ }_{2} F_{1}$ the hypergeometric function, which holds when $a \leq b \leq c$.

The $a, b$ and $c$ are the exacts roots of a third order polynomial and are thus awkward expressions even for our simple unperturbed problem. Surprisingly, the combinations $b-a$ and $c-a$ reduce to manageable expressions:

$$
\begin{aligned}
& b-a=\sqrt{3} \sin \left(\frac{1}{3} \arcsin (12 E-1)+\frac{\pi}{6}\right) \\
& c-a=\sqrt{3} \cos \left(\frac{1}{3} \arcsin (12 E-1)\right)
\end{aligned}
$$

Substituting all this we get

$$
\begin{aligned}
\int y_{2} d y_{1}= & 2 \frac{1}{24} \sqrt{6} \pi 3 \sin ^{2}\left(\frac{1}{3} \arcsin (12 E-1)+\frac{\pi}{6}\right) \times \\
& \times \sqrt{\sqrt{3} \cos \left(\frac{1}{3} \arcsin (12 E-1)\right)} \times \\
& \times{ }_{2} F_{1}\left(-\frac{1}{2}, \frac{3}{2}, 3, \frac{\sin \left(\frac{1}{3} \arcsin (12 E-1)+\frac{\pi}{6}\right)}{\cos \left(\frac{1}{3} \arcsin (12 E-1)\right)}\right)
\end{aligned}
$$

The factor 2 arises because we have to integrate once from $b$ to $a$ and once from $a$ to $b$.

To calculate the period of the periodic orbit of the unperturbed system, we apply the standard technique of separation of variables to the exact integral (2.7). This leads us through a calculation similar to the one above, resulting in:

$$
\begin{aligned}
\text { period }= & 2 \sqrt{6} \frac{1}{\sqrt{\sqrt{3} \cos \left(\frac{1}{3} \arcsin (12 E-1)\right)}} \times \\
& \times K\left(\frac{\sin \left(\frac{1}{3} \arcsin (12 E-1)+\frac{\pi}{6}\right)}{\cos \left(\frac{1}{3} \arcsin (12 E-1)\right)}\right)
\end{aligned}
$$

where $\mathrm{K}$ is the complete elliptic integral of the first kind.

We finally obtain the averaged equation by dividing equation (3.6) by equation (3.7) and adding some extra factors from equation (3.1):

$$
\begin{aligned}
\dot{\bar{E}} & =2 \epsilon \frac{a^{\prime}(\epsilon t)}{a(\epsilon t)} \frac{\int y_{2} d y_{1}}{\text { period }} \\
& =\epsilon \frac{a^{\prime}(\epsilon t)}{a(\epsilon t)} A(\bar{E})
\end{aligned}
$$

It does not add much to the understanding of the problem to write down the averaged equation in it's full form. That is why we omit this. All that matters is that the right hand side is an explicitly known function $A(\bar{E})$ of $\bar{E}$, which we can approximate to arbitrary precision, and of time.

We note that the averaged energy equation (3.8) is in the form

$$
\frac{\mathrm{d} \bar{E}}{\mathrm{~d} T}=-\frac{1}{2 \pi} \omega(\bar{E}) D(\bar{E}, T)
$$

where $T=\epsilon t, \omega(\bar{E})$ is the frequency of the nonlinear oscillator and $\epsilon D(\bar{E}, T)$ is the change in energy over one period (including slow variations and dissipation). This dissipation function $D$ approaches the Melnikov integral as $\bar{E}$ approaches its critical value associated with the homoclinic orbit $\left(\frac{1}{6}\right)$. 
3.1.2. Validity of the averaged equation. Since the averaged equation is an approximation of the exact system (2.2), we have to address the question of the accuracy of this approximation, on which timescale it holds and where in the stable region.

We expect that the closer we start to the homoclinic orbit of the unperturbed system, the less accurate the averaged equation will become. The dynamics splits up in two qualitatively different time intervals, the first in which the orbit slowly separates from the homoclinic orbit and the second in which the orbit slowly spirals towards the attracting origin.

We start with the first time-interval. As we will show in section 3.2, apart from a sub-boundary layer of size $O\left(\exp \left(-\frac{1}{\epsilon}\right)\right)$, this time-interval has a size of $O\left(\frac{1}{\epsilon}\right)$ independent of the initial distance from the homoclinic orbit. The total error introduced by the averaging process in the first time-interval is of $O\left(\epsilon T_{0}\right)\left(T_{0}\right.$ is the period of the unperturbed orbit corresponding to $\bar{E}(0)$, the initial value of $\bar{E})$. A short explanation of this estimate is given in section 5 .

For the second interval we can make use of the standard averaging theorems, from which we get that the introduced error on the second interval is of $O(\epsilon)$ and that we are allowed to extend the second interval to infinity, because all orbits are attracted to the origin (see Sanders en Verhulst [19], chapter 4).

This attracting property of the orbits also implies that the error introduced from the first does not blow up. Therefore, the total error introduced by the averaging process is of $O\left(\epsilon T_{0}\right)$ valid for all time. As we will also show in section 3.2, for orbits starting close to the homoclinic orbit of the unperturbed system $\bar{E}(0)=\frac{1}{6}$, we have that $T_{0}$ is of order $-\log \left(\frac{1}{6}-\bar{E}(0)\right)$, which implies that the averaged equation can be used to approximate the dynamics up to a distance of $O\left(\exp \left(-\frac{1}{\epsilon}\right)\right)$ from the homoclinic orbit of the unperturbed system. More quantitative details on the boundary layer will be given in section 3.2. We will also see in section 3.2 that the averaged equation indeed breaks down when we approach the boundary layer.

3.1.3. Analysis of the averaged equation. We now turn to the analysis of the averaged equation (3.8). The first thing one should notice is that the effect of the decaying function $a(\epsilon t)$ can be removed from the equation by transforming to the new time $\tau$

$$
\left\{\begin{aligned}
\tau & =-\frac{1}{\epsilon} \log (a(\epsilon t)) \\
a(\epsilon t) & =e^{-\epsilon \tau}
\end{aligned}\right.
$$

Note that this transformation reduces to the identity transformation in the special case $a(\epsilon t)=e^{-\epsilon t}$.

It is remarkable that, given condition (3.2), it is not important at all how $a(\xi)$ decays to zero, the dynamics of the system does not change, apart from a rescaling of the time axis.

Applying this transformation produces the autonomous, 1-dimensional system

$$
\frac{\mathrm{d} \bar{E}}{\mathrm{~d} \tau}=-\epsilon A(\bar{E})
$$

We can solve this system explicitly by separation of variables, but unfortunately we do not have a primitive of $\frac{1}{A(\bar{E})}$ in the form of an elementary function.

But we can draw some important conclusions from this system, of which the most 

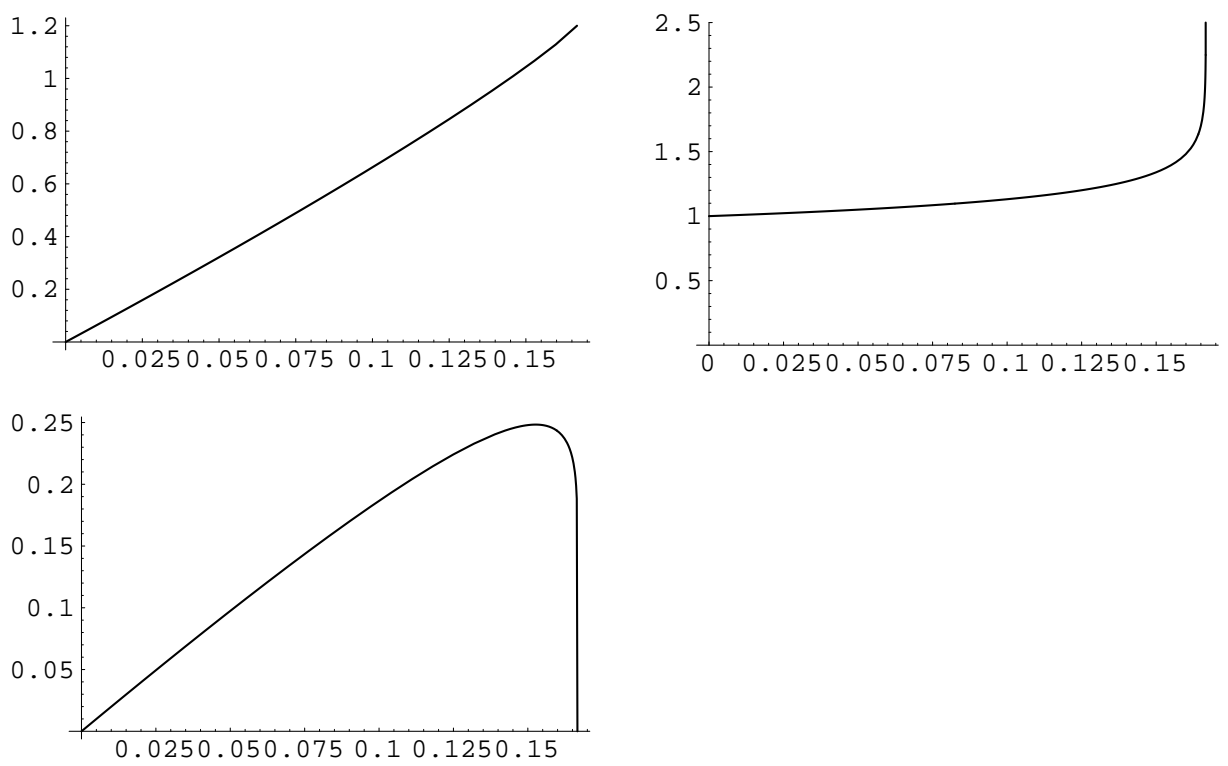

FIG. 3.1. The dependence of $\int y_{2} d y_{1}$, the $\frac{\text { period }}{2 \pi}$ and $A$ on $\bar{E}$

important one is the existence of an adiabatic invariant: As noted, it is always possible to solve system (3.11), which gives the solution $\bar{E}=\bar{E}(\bar{E}(0), \epsilon \tau)$ as a function of the initial condition and slow time. Again, in principle one can solve this equation for $\bar{E}(0)$ as a function of $\bar{E}$ and $\epsilon \tau$. Inverting the time transformation (3.10), one finds $\bar{E}(0)$ as a function of $\bar{E}$ and $\epsilon t$. Since $\bar{E}(0)$ is obviously time-independent, we reach the conclusion that

There exists a global adiabatic invariant in side the homoclinic orbit of the unperturbed system with the exclusion of an exponentially thin boundary layer, valid for all time, determined by equation (3.11).

For special cases we are able to produce these calculations explicitly, which we will show now. To understand these cases well, it is important to know how $\int y_{2} d y_{1}$, the period and $A$ depend on $\bar{E}$. This is shown in figure 3.1 .

It is clear that $\int y_{2} d y_{1}$ depends almost linearly on $\bar{E}$ throughout the entire interval. This is understandable, since it is similar to the dependence of the area of a disk on its radius. What is not transparent is that the derivative of this function goes to infinity as $\bar{E}$ goes to $\frac{1}{6}$, but so slowly that its integral remains bounded.

The period is close to $2 \pi$ for small $\bar{E}$ as it should be, because in this region the unperturbed system behaves nearly like a harmonic oscillator with $\omega=1$. When $\bar{E}$ goes to $\frac{1}{6}$, the period goes to infinity, because the orbits are approaching the saddle point, in the neighbourhood of which they will stay a long time for each passage.

The quotient of the two, $A$, shows the linear behaviour of $\int y_{2} d y_{1}$ for small $\bar{E}$, because the period is almost constant. However, $A$ has a maximum $(0.248320 \ldots)$ at $\bar{E}=0.152640 \ldots$, after which it rapidly drops to zero. We could have predicted that $A$ is small for $\bar{E}$ close to $\frac{1}{6}$, since all the time the orbits are close to the saddle point, the righthand side of equation $(3.1)$ is small $\left(y_{2} \ll 1\right)$, resulting in a small average. 
3.1.4. The adiabatic invariant. We now turn to the calculation of the adiabatic invariant for $\bar{E}(0)$ small (we will make this more precise later on).

To approximate the adiabatic invariant, we perform a Taylor expansion of $A(\bar{E})$ around 0 . We note that the hypergeometric function forces us to use $\sqrt{E}$ as expansion variable instead of just $\bar{E}$. However, it turns out that the coefficients in front of the non-integer powers of $\bar{E}$ are equal to zero, at least to fifth order. After a long calculation we arrive at the following expansion, valid for $0 \leq \bar{E}<\frac{1}{6}$

$$
A(\bar{E})=2 \bar{E}-\frac{5}{6} \bar{E}^{2}-\frac{155}{54} \bar{E}^{3}-\frac{61135}{5184} \bar{E}^{4}-\frac{825409}{15552} \bar{E}^{5}+O\left(\bar{E}^{5 \frac{1}{2}}\right)
$$

To approximate the adiabatic invariant, we truncate the series after the second order terms, since we are interested in the first non-trivial deviation from a slowly attracting focus. Substituting this quadratic expression into the averaged equation (3.11) we get

$$
\frac{\mathrm{d} \bar{E}}{\mathrm{~d} \tau}=-2 \epsilon \bar{E}+\frac{5}{6} \epsilon \bar{E}^{2}
$$

which is easy to solve giving

$$
\bar{E}(\epsilon \tau)=\frac{2 \bar{E}(0)}{\left(2-\frac{5}{6} \bar{E}(0)\right) e^{2 \epsilon \tau}+\frac{5}{6} \bar{E}(0)}
$$

From this we readily obtain the adiabatic invariant

$$
\frac{\bar{E}(0)}{2-\frac{5}{6} \bar{E}(0)}=\frac{\bar{E}(\epsilon \tau)}{2-\frac{5}{6} \bar{E}(\epsilon \tau)} e^{2 \epsilon \tau}
$$

We are now able to specify what we meant with $\bar{E}(0)$ small. Since we have neglected $O\left((\bar{E}(0))^{3}\right)$ terms, we have introduced a new error of order $(\bar{E}(0))^{3}$ in the approximation of the solution. Since we do not want this error term to dominate the error introduced by the averaging process $(O(\epsilon))$, we take $\bar{E}(0)$ to be $O\left(\epsilon^{1 / 3}\right)$.

Expanding the adiabatic invariant around $\bar{E}=0$, we see that the first non-trivial correction to the slowly attracting focus (with adiabatic invariant $\bar{E}(0)=\bar{E} e^{2 \epsilon \tau}$ ) is given by $\frac{5}{48} \bar{E}^{2} e^{2 \epsilon \tau}$ resulting in a slightly slower collapse onto the origin $\left(y_{1}, y_{2}\right)=$ $(0,0)$.

These arguments hold for the $\left(y_{1}, y_{2}\right)$ phase space only. To extend them to the original $\left(x_{1}, x_{2}\right)$ phase space, we have to invert the time-transformation (3.10) and the phase space transformation (2.1), after which we obtain the adiabatic invariant in the $\left(x_{1}, x_{2}\right)$ phase space:

$$
\frac{3 a(\epsilon t) x_{1}^{2}-2 a(\epsilon t)^{2} x_{1}^{3}+6 \epsilon a^{\prime}(\epsilon t) x_{1} x_{2}+3 a(\epsilon t) x_{2}^{2}}{72 a(\epsilon t)-15 a(\epsilon t)^{3} x_{1}^{2}+10 a(\epsilon t)^{4} x_{1}^{3}-30 \epsilon a(\epsilon t)^{2} a^{\prime}(\epsilon t) x_{1} x_{2}-15 a(\epsilon t)^{3} x_{2}^{2}}
$$

We include this rather lengthy expression, because it reveals an important phenomenon: Due to the cross-terms $x_{1} x_{2}$, the level curves of the adiabatic invariant for a fixed time "resemble" ellipses, of which the long axis and the short axis differ by an $O(\epsilon)$ 


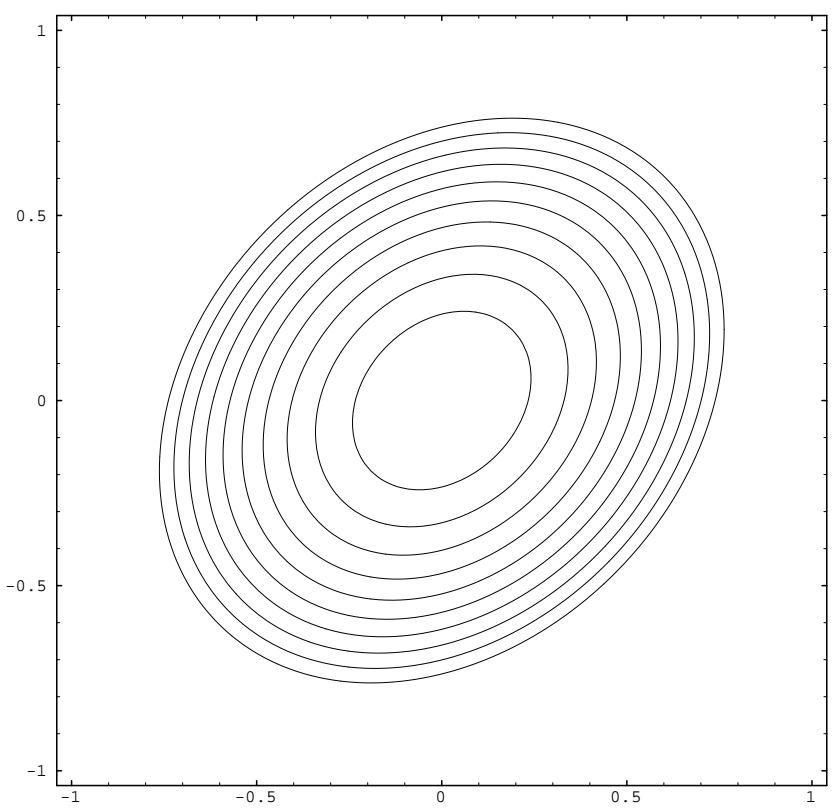

Fig. 3.2. A few level curves of the adiabatic invariant for $t$ fixed at infinity

amount. and which are rotated around the origin, causing asymmetry with respect to the $y_{1}$ and $y_{2}$ axis.

We did expect this for finite time, but this behaviour persists when we let $t$ tend to infinity. Put in other words, when $t$ goes to infinity, our dynamical system (1.3) becomes symmetric (with respect to $x_{1}$ and $x_{2}$ ), but the level curves of the adiabatic invariant remain asymmetric. We have reached this important conclusion:

The evolution of an ensemble of phase points towards a symmetric potential will show significant (i.e. $O(\epsilon))$ traces of its asymmetrical past, for all time.

So there is a sort of hysteresis effect present: although the system becomes symmetric, it still "knows" that it was asymmetric in the past.

We note that this phenomenon is not present in the $\left(y_{1}, y_{2}\right)$ phase space, where the level curves of the adiabatic invariant are symmetric with respect to the $y_{1}$-axis, but is introduced by the phase space transformation (2.1) alone.

To demonstrate this phenomenon visually, we have to take $\epsilon$ not too small, so we took $\epsilon=\frac{1}{4}$. Figure 3.2 shows a few level curves of the adiabatic invariant for $a(\epsilon t)=e^{-\epsilon t}$ and $t$ fixed at infinity. The asymmetric effect is clearly present.

As explained before, we expected to see effects of the slowly decaying asymmetry in the neighbourhood of the boundary layer separating the stable and unstable region, but now it turns out that there are effects $(O(\epsilon))$ close to the origin too.

3.2. Approaching the boundary layer. We study the approach to the boundary layer, which is an $o(1)$ domain near the homoclinic orbit and the stable manifold.

More precisely, the boundary layer can be divided into three regions (see figure $3.3)$. The first region consists of the phase points which are between $O\left(\exp \left(-\frac{1}{\epsilon}\right)\right)$ and $o(1)$ inside the homoclinic orbit of the unperturbed system. It is in this region that the averaging technique slowly loses its validity, as explained in section 5 . We will 


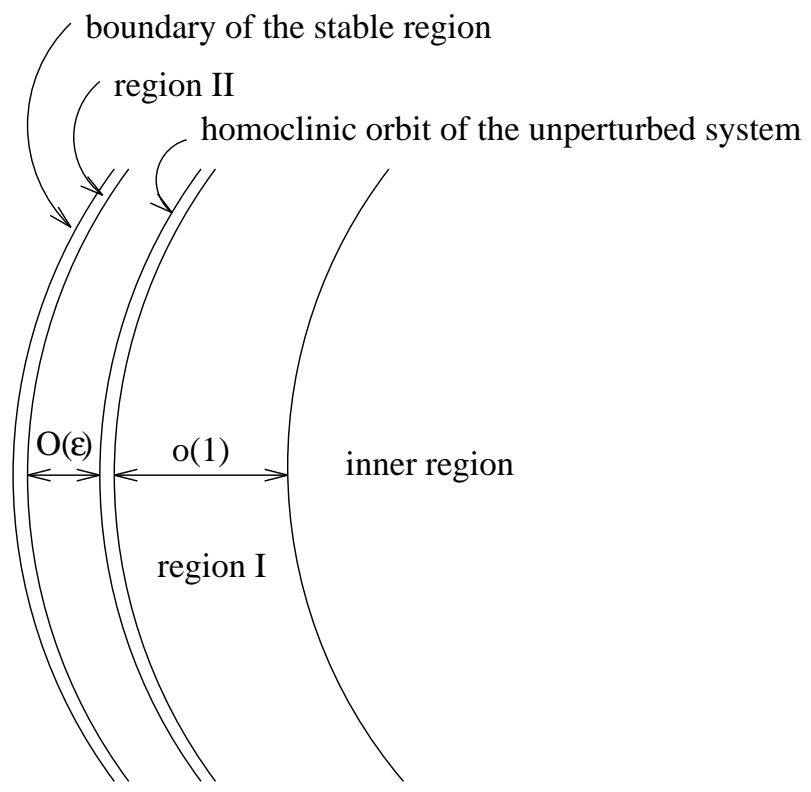

Fig. 3.3. The structure of the boundary layer

call this region the o(1) boundary layer.

The second region consists of the phase points which are within an $O\left(\exp \left(-\frac{1}{\epsilon}\right)\right)$ neighbourhood of the boundary of the stable region. Orbits starting in these points will pass the saddle point $\left(y_{1}, y_{2}\right)=(1,0)$ on at least a $\frac{1}{\epsilon}$ timescale (which requires special attention), after which they will enter the third region. We will call this region the $O\left(\exp \left(-\frac{1}{\epsilon}\right)\right)$ boundary layer.

The third region consists of the remaining phase points in the boundary layer, which is a strip with an $O(\epsilon)$ width. Orbits starting in this region will enter the first region on an $O(1)$ timescale, which allows us to use the unperturbed orbits inside this region. We will call this region the $O(\epsilon)$ boundary layer.

The inner region, finally, consists of the phase points inside the stable region but outside the boundary layer.

Using the same approach as in the previous subsection, we are able to study the adiabatic invariant everywhere in the inner region and the $o(1)$ boundary layer. The general idea is to expand the averaged equation around a certain value of $\bar{E}$, in the neighbourhood of which we want to study the adiabatic invariant. This can be done to any desired precision. For low order expansions, it is possible to integrate the resulting equation explicitly. For high orders, one has to use numerical methods.

Approaching the boundary layer, there are two more special values of $\bar{E}$ to study, knowing the value of $\bar{E}$ corresponding to the maximum of figure (3.1) and the maximum value $\bar{E}=\frac{1}{6}$.

The study in the neighbourhood of the local maximum $\bar{E}_{\max }=0.1526396 \ldots$ has restricted mathematical and physical value, which is why we do not include it here. The calculations are straightforward, leading to an expression for the adiabatic invariant involving the artanh function.

The second special value of $\bar{E}, \frac{1}{6}$, is much more interesting. Expanding the averaged equation around $\frac{1}{6}$ is not simple, because the hypergeometric function has 
an infinite derivative at this point, and the elliptic integral (the period) is unbounded at this point.

One should also keep in mind that the averaged equation is not valid exponentially close (with respect to $\epsilon$ ) to the unperturbed homoclinic orbit. Therefore, the results of expanding the averaged equation around $\frac{1}{6}$ is valid close (with respect to $\bar{E}$ ) but not exponentially close (with respect to $\epsilon$ ) to the unperturbed homoclinic orbit.

We break up this calculation by expanding equation (3.6) and equation (3.7) separately. After a straightforward calculation, we arrive at the following expansions:

$$
\begin{gathered}
\int y_{2} d y_{1}=\frac{6}{5}-72\left(1-\log \left(\frac{\frac{1}{6}-\bar{E}}{72}\right)\right)\left(\frac{\frac{1}{6}-\bar{E}}{72}\right) \\
+O\left(\log \left(\frac{1}{6}-\bar{E}\right)\left(\frac{1}{6}-\bar{E}\right)^{2}\right) \\
\text { period }=-\log \left(\frac{\frac{1}{6}-\bar{E}}{72}\right)-12\left(26+5 \log \left(\frac{\frac{1}{6}-\bar{E}}{72}\right)\right)\left(\frac{\frac{1}{6}-\bar{E}}{72}\right) \\
+O\left(\log \left(\frac{1}{6}-\bar{E}\right)\left(\frac{1}{6}-\bar{E}\right)^{2}\right)
\end{gathered}
$$

Substituting these two expansions, we obtain for the averaged equation (with $O\left(\epsilon\left(\frac{1}{6}-\bar{E}\right)^{2}\right)$ terms neglected $)$

$(3.19) \frac{\mathrm{d} \bar{E}}{\mathrm{~d} \tau}=\epsilon \frac{12}{5 \log \left(\frac{\frac{1}{6}-\bar{E}}{72}\right)}+144 \epsilon\left(1-\frac{2}{\log \left(\frac{\frac{1}{6}-\bar{E}}{72}\right)}-\frac{26}{5 \log ^{2}\left(\frac{\frac{1}{6}-\bar{E}}{72}\right)}\right)\left(\frac{\frac{1}{6}-\bar{E}}{72}\right)$

This equation is too complicated to be solved analytically. However, if we neglect the $O\left(\epsilon\left(\frac{1}{6}-\bar{E}\right)\right)$ term too, it is again possible to calculate the adiabatic invariant explicitly:

$$
I_{\bar{E}_{1 / 6}}=\left(\frac{\frac{1}{6}-\bar{E}}{72}\right) \log \left(\frac{\frac{1}{6}-\bar{E}}{72}\right)-\left(\frac{\frac{1}{6}-\bar{E}}{72}\right)+\frac{1}{30} \epsilon \tau
$$

Note that this adiabatic invariant is only valid on an $\frac{1}{\sqrt{\epsilon}}$ timescale, since $\frac{1}{6}-\bar{E}$ will become $O(\sqrt{\epsilon})$ on this timescale, causing an extra error of $O(\epsilon)$.

At this point we are able to make some important remarks:

- Every orbit starting inside the $o(1)$ boundary layer will collapse onto the attracting focus $\left(y_{1}, y_{2}\right)=(0,0)$ on an $\frac{1}{\epsilon}$ timescale, independent of the initial distance from the homoclinic orbit (collapsing onto the origin in the $\left(y_{1}, y_{2}\right)$ plane is equivalent to circling around the origin in the $\left(x_{1}, x_{2}\right)$ plane). This follows directly from the adiabatic invariant (3.20), which forces the orbits away from the boundary layer on an $\frac{1}{\epsilon}$ timescale.

- The averaging process breaks down in the small strip between the $o(1)$ boundary layer and the homoclinic orbit of the unperturbed system, like we expected it to. If the averaging process would be valid there too, every orbit starting there would collapse onto $\left(y_{1}, y_{2}\right)=(0,0)$ on an $\frac{1}{\epsilon}$ timescale, which would imply that all these orbits stay within a certain bounded neighbourhood of the origin in the $\left(x_{1}, x_{2}\right)$ plane. This cannot be true of course, because an orbit starting very close to the saddle point $\left(x_{1}, x_{2}\right)=(1,0)$ inside the homoclinic orbit, will end up arbitrarily far away from the origin in the $\left(x_{1}, x_{2}\right)$ plane. 
- The leading order behaviour of the period near the homoclinic orbit is given by $-\log \left(\frac{\frac{1}{6}-\bar{E}}{72}\right)$. This is the cause of the break-down of the averaging process, since averaging is only valid if the period is $o\left(\frac{1}{\epsilon}\right)$.

- The applicability of averaging near the homoclinic orbit is limited to calculating energy-differences. Since energy-differences are our main interest, we do not need any other mathematical tools in our study. A more general treatment, including the calculation of time- and phase-differences near the homoclinic orbit, can be found e.g. in Tennyson, Cary and Escande [20] and Bourland and Haberman [2].

4. The boundary layer. After the previous study of the major part of the stable region, we will turn our attention to the remaining part of the boundary layer. Since the $o(1)$ boundary layer is covered by the previous section, we only have to study the $O(\epsilon)$ and $O\left(\exp \left(-\frac{1}{\epsilon}\right)\right)$ boundary layers. As we explained in the previous section, we cannot use the theory of averaging for this study.

We treat the $O(\epsilon)$ and $O\left(\exp \left(-\frac{1}{\epsilon}\right)\right)$ boundary layers simultaneously. The only difference between them is that orbits starting inside the $O\left(\exp \left(-\frac{1}{\epsilon}\right)\right)$ boundary layer will pass the the saddle point $\left(y_{1}, y_{2}\right)=(1,0)$ on at least a $\frac{1}{\epsilon}$ timescale, which results in an arbitrary large circle in the $\left(x_{1}, x_{2}\right)$ plane as t tends to infinity. However this does not require a separate treatment.

It is important to note that orbits starting inside the $O(\epsilon)$ boundary layer will remain within an $O(1)$ neighbourhood of the origin in the $\left(x_{1}, x_{2}\right)$ plane. So, although the $O(\epsilon)$ boundary layer has a larger thickness than the $O\left(\exp \left(-\frac{1}{\epsilon}\right)\right)$ boundary layer, it is in fact much smaller, because the latter has to fill up the rest of the $\left(x_{1}, x_{2}\right)$ phase space.

To study the boundary layer, we can use the same method we used to compute the position of the boundary of the stable region, because the orbits in the boundary layer are close to the homoclinic orbit of the unperturbed problem. So, to calculate the orbits to $O(\epsilon)$ precision, we are allowed to substitute expressions for the homoclinic orbit into the $O(\epsilon)$ contributions to the dynamics.

This way we get again a two stage process. The first stage is governed by the homoclinic orbit of the unperturbed system. After the orbit has entered the domain of validity of the averaging process, the orbit collapses onto the origin on a $\frac{1}{\epsilon}$ timescale.

4.1. An adiabatic invariant inside the boundary layer. Since the existence of an adiabatic invariant was of great help in our study of the inner region, we prefer to extend that approach to the boundary layer. We expect an adiabatic invariant to be present in the boundary layer too, because we are studying a Hamiltonian system which depends adiabatically on time. Finding this adiabatic invariant is generally very hard in regions where the unperturbed system has non-periodic solutions (in our case, outside the homoclinic orbit).

The straightforward way to find the adiabatic invariant is to perturb the energy of the unperturbed system in such a way that its time-derivative becomes $O\left(\epsilon^{2}\right)$. So we are looking for an adiabatic invariant of the form

$$
I_{b l}\left(y_{1}, y_{2}, \epsilon t\right)=E(\epsilon=0)+\epsilon g\left(E(\epsilon=0), y_{1}, \epsilon t\right)
$$

where $E(\epsilon=0)$ is given by $(2.7)$.

By demanding that the time-derivative of $I_{b l}$ has a zero $O(\epsilon)$ contribution, one normally arrives at a first order linear PDE for the function $g$. With a little bit of 
foresight, we choose the first argument to be orthogonal to the characteristic lines of the PDE, which is why we arrive at a first order linear ODE for the function g.

By using Gradshteyn and Ryzhik [6] intensively we derived this explicit expression for the function $g$ :

$$
\begin{aligned}
g(E(\epsilon= & \left.0), y_{1}, \epsilon t\right)=-\frac{8}{15} \sqrt{\frac{2}{3}} \frac{a^{\prime}(\epsilon t)}{a(\epsilon t)} \times \\
& \times\left\{\left(-b \xi+\frac{3}{2} \xi^{3}\right) \sqrt{\left(\xi^{2}-b\right)^{2}+c^{2}}+\left(b^{3}+b c^{2}\right) I_{1}-\frac{9}{4} I_{2}\right\} \\
I_{1}= & \frac{1}{2}\left(b^{2}+c^{2}\right)_{2}^{-1 / 4} F_{1}(\alpha, r) \\
I_{2}= & \frac{1}{2}\left(b^{2}+c^{2}\right)^{1 / 4}\left({ }_{2} F_{1}(\alpha, r)-2 E(\alpha, r)\right)+\frac{\sqrt{\left(\xi^{2}-b\right)^{2}+c^{2}}}{\xi+\sqrt{b^{2}+c^{2} \xi^{-1}}} \\
\alpha= & \arccos \left\{\frac{\sqrt{b^{2}+c^{2}}-\xi^{2}}{\sqrt{b^{2}+c^{2}}}+\xi^{2}\right. \\
r= & \frac{1}{2}\left(1+\frac{b}{\sqrt{b^{2}+c^{2}}}\right)
\end{aligned}
$$

Note that $E(\alpha, r)$ is the elliptic integral of the second kind and not the energy. The real numbers $a, b$ and $c$ are related to the roots of a third order polynomial in this way

$$
2 E(\epsilon=0)-y_{1}^{2}+\frac{2}{3} y_{1}^{3}=\frac{2}{3}\left(y_{1}-a\right)\left(\left(y_{1}-a-b\right)^{2}+c^{2}\right) \quad \forall y_{1}
$$

So $a, b$ and $c$ are functions of $E(\epsilon=0)$, with $a<0, b \geq 0$ and $c \geq 0$. We want to make five remarks with respect to this formula:

- We have derived the adiabatic invariant outside the homoclinic orbit (but inside the stable region) of the unperturbed system. It is however not possible to calculate the adiabatic invariant in the inner region using the same procedure, since the characteristic lines are closed curves in the inner region, which prohibits the PDE to have a solution. Indeed, the adiabatic invariant we found previously for the inner region has an $O(\epsilon)$ time-derivative.

- $I_{b l}$ determines the dynamics inside the boundary layer completely. This follows easily from $d\left(I_{b l}\right)=0$.

- $I_{b l}$ is symmetric in $y_{2}$. Transforming back to the $\left(x_{1}, x_{2}\right)$ plane introduces again the cross-terms $x_{1} x_{2}$ in the adiabatic invariant which do not vanish for $t$ going to infinity.

- We now have an adiabatic invariant throughout the entire stable region, with the exception of the very thin $\left(O\left(\exp \left(-\frac{1}{\epsilon}\right)\right)\right)$ region between the homoclinic orbit of the unperturbed system and the $o(1)$ boundary layer. This is not a problem, since we can approximate the dynamics inside this strip with transversal orbits which introduces only an $O\left(\frac{1}{\epsilon} \exp \left(-\frac{1}{\epsilon}\right)\right)$ error. This trick solves the problem of matching the two adiabatic invariants at the same time.

- Orbits starting very close ("closer than exponentially close") do not reach the homoclinic orbit on a $\frac{1}{\epsilon}$ timescale, and are therefore not covered by this treatment. In the original coordinates, these orbits simply travel together with the saddle-point towards infinity and are thus not very interesting.

4.2. Numerical study of the dynamics inside the boundary layer. We would like to visualize the dynamics going on inside the boundary layer. Density functions are not very useful for this, since our system is Hamiltonian which implies 


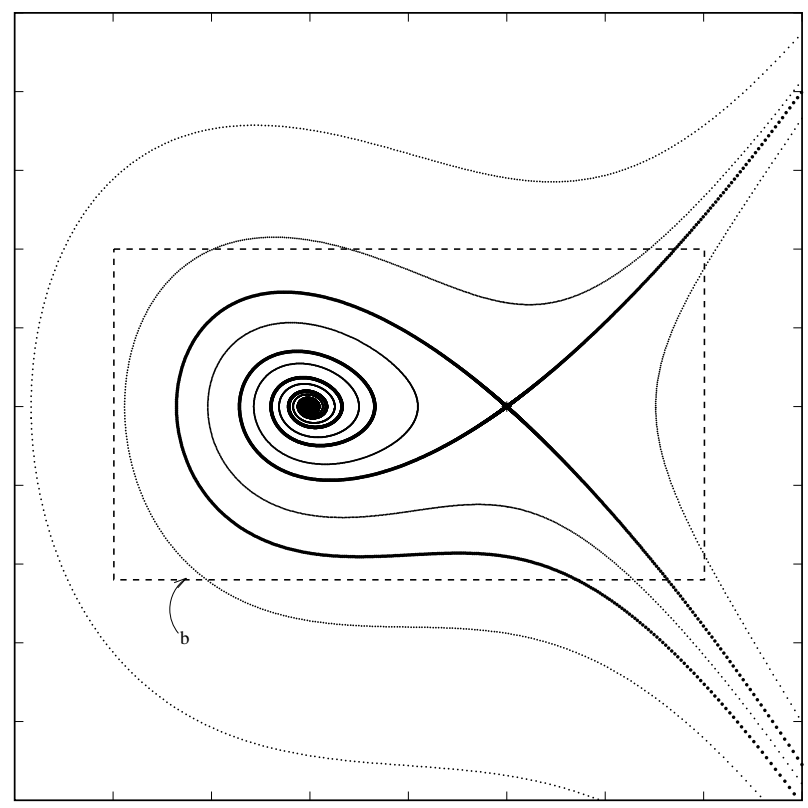

FIG. 4.1. The rectangular box around the homoclinic orbit of the unperturbed system.

area conservation. This is well-known for autonomous and time-periodic Hamiltonian systems. To prove it for general time-dependent systems, one introduces a new independent variable equal to the time (making the system autonomous). Applying Liouville's theorem proves the desired result.

Note that the conservation of area implies that the area of the "tongue" of the boundary layer is infinite, since it has to fill up the entire $\left(x_{1}, x_{2}\right)$ phase space in the end. So our "thin" boundary layer is in fact the largest part of the stable region.

To study the dynamics inside the boundary layer, we therefore choose to look at the evolution of the rectangular box around the homoclinic orbit of the unperturbed system, as depicted in figure 4.1 .

Since all orbits starting inside the box will remain inside the (evoluted) box, we only have to study the boundary of the box. Moreover, we only have to study those points of the boundary lying inside the stable region, since all other points clear off to infinity on an $O(1)$ timescale. Therefore we only have to study the bottom boundary (b) of the box.

So by studying only a very limited set of phase space, we will gain information about all orbits starting inside the box, i.e. both inside the domain of validity of averaging and inside the boundary layer.

For numerical reasons, we followed the evolution of the bottom boundary of a different (but similar) box in the $\left(x_{1}, x_{2}\right)$ phase space, namely the straight line between $\left(x_{1}, x_{2}\right)=(0,-2.5)$ and $\left(x_{1}, x_{2}\right)=(5,-2.5)$. The numerical results are shown in figure 4.2. We took $\epsilon=0.1$ which forced us to take steps along the boundary as small as $10^{-14}$ to generate the last sub-figure. This is due to the fact that the most interesting dynamics takes place in an $O\left(\exp \left(-\frac{1}{\epsilon}\right)\right)$ neighbourhood of the boundary of the stable region.

The open area around the origin is the domain of validity of averaging. This is the part of phase space where the level curves of the adiabatic invariant (figure 3.2) live. 

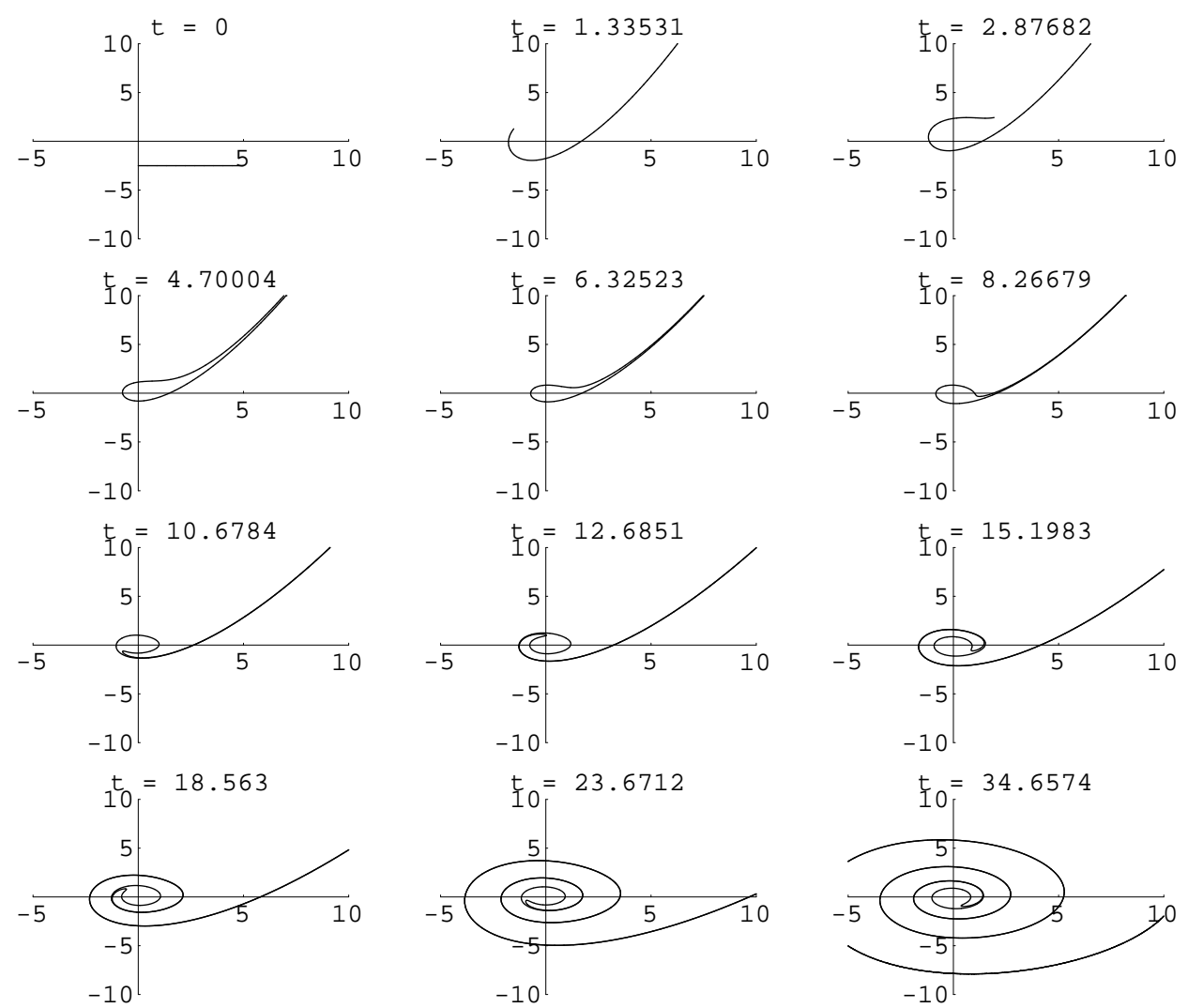

FIG. 4.2. The evolution of a straight line (part of the bottom boundary b) crossing the boundary of the stable region.

It is also clear to see the instantaneous saddle point moving from $(1,0)$ to infinity.

The points connecting the instantaneous saddle point with the domain of validity of averaging have started very close $\left(O\left(\exp \left(-\frac{1}{\epsilon}\right)\right)\right)$ to the boundary of the stable region, passed the saddle point during a time-interval of $O\left(\frac{1}{\epsilon}\right)$, after which they entered the domain of validity of averaging (in the $\left(y_{1}, y_{2}\right)$ phase space).

The effect in the $\left(x_{1}, x_{2}\right)$ phase space is that the orbits end up circling around the origin outside the part where the level curves of the adiabatic invariant (figure 3.2) live. The closer an orbit starts to the boundary of the stable region, the larger the radius of the circle it describes in the end.

The effect of the area conservation is also nicely visible. Since the starting box has a finite area, the area inside the spiral must be finite too, which makes the spiral very thin. Note that from $t=4.7$ on the curve going to infinity actually consists of two very close curves.

Note also that the remaining (major) part of phase space has to be filled by the tail of the "small" tongue of the boundary layer which lies outside the box.

4.3. Estimation of the time-difference between the first two saddle approaches. A physically very relevant quantity is the time-difference between the saddle approaches just before and just after the orbit has crossed the homoclinic orbit. We note that these are the only two important saddle approaches in our problem. Before an orbit crosses the homoclinic orbit, it can pass the saddle only once, and 
after passing the saddle for the second time (i.e. after crossing the homoclinic) the orbit will lie in the domain of phase space where averaging methods are applicable.

Once an orbit has entered the domain of averaging, it will soon start spiralling around the origin in the $\left(y_{1}, y_{2}\right)$ phase space, resulting in a circular motion in the original $\left(x_{1}, x_{2}\right)$ phase space. To compute the radius of this circular motion, we need to know the location of the saddle point when the orbit enters the domain of averaging, and thus the time-difference between the first two saddle approaches. Using this as the initial condition for the adiabatic invariant valid inside the domain of averaging, one easily obtains the unknown radius.

By expanding the explicitly known adiabatic invariant valid inside the boundary layer (4.2) near the boundary of the stable region, we could derive the first approximation to the time-difference between the first two saddle approaches.

Instead we use a nice result derived by Bourland and Haberman [2], which gives us the desired expression for the time-difference between the first two saddle approaches after a rather straightforward calculation:

$$
\Delta t=-\log \left(\frac{1}{72} \sqrt{W_{0}\left(W_{0}-\frac{12}{5} \frac{a^{\prime}(0)}{a(0)} \epsilon\right)}\right)
$$

with $W_{0}$ the distance (in energy) between the saddle-point and the orbit at the first saddle-approach after crossing the homoclinic orbit.

This result shows again that in order to move an arbitrary $O(1)$ distance away from the origin in the $\left(x_{1}, x_{2}\right)$ phase space, an orbit must start exponentially close to the boundary of the stable region. Only then the time-difference (4.4) will be of order $\frac{1}{\epsilon}$ which produces an $O(1)$ displacement away from the origin in the $\left(x_{1}, x_{2}\right)$ phase space. Smaller time-differences (typically $O(\log \epsilon)$ ) can only have an $o(1)$ effect in the $\left(x_{1}, x_{2}\right)$ phase space and are thus less interesting in this study.

5. Concluding remarks. It is surprising that it is possible to give a fairly complete treatment of system (1.3) which describes the evolution of a simple system with an asymmetric potential to a symmetric potential. The most remarkable result is that in the evolution towards symmetry as time tends to infinity, traces of the asymmetric past can be recognized in the solutions.

System (1.3) is just a metaphor for simplified models with two degrees of freedom which exhibit evolution from asymmetry towards symmetry. In a forthcoming paper we shall discuss such higher dimensional problems using basically the same methods.

There are two interesting variants of our model problem (1.3) which need special attention. In the first one, the function $a$ is taken to decay linearly, which clearly does not satisfy the condition that $\frac{a^{\prime}}{a}$ must remain bounded. In the second one, the limiting value of $a$ is chosen differently from zero, which avoids the movement of the saddle-point to infinity.

In the discussion of the validity of the averaged equation, we have assumed that the reader is familiar with the proof of the standard averaging theorems (see for instance Sanders and Verhulst [19]). In particular, we have used the straightforward extension of those theorems to periods depending on $\epsilon$ : by rescaling the time-variable it is easily shown that averaging produces $O(\epsilon T(\epsilon))$ asymptotic approximations, valid on a $\frac{1}{\epsilon}$ timescale, as long as $\epsilon T(\epsilon)$ is $o(1)$.

6. Acknowledgement. We would like to thank prof. Richard Haberman, who provided us with useful suggestions, questions and references. 


\section{REFERENCES}

[1] J. Binney and S. Tremaine, Galactic dynamics, Princeton series in astrophysics, Princeton University Press, 41 William Street, Princeton, New Jersey 08540, 3rd ed., 1994.

[2] F. Bourland and R. Haberman, Separatrix crossing: Time-invariant potentials with dissipation, SIAM J. Appl. Math., 50 (1990), pp. 1716-1744.

[3] - Connection across a separatrix with dissipation, Studies in Appl. Math., 91 (1994), pp. 95-124.

[4] F. Bourland, R. Haberman, And W. Kath, Averaging methods for the phase shift of arbitrarily perturbed strongly nonlinear oscillators with an application to capture, SIAM J. Appl. Math., 51 (1991), pp. 1150-1167.

[5] J. Cole, Perturbation methods in applied mathematics, Blaisdell, Waltham, Massachusetts, 1968.

[6] I. Gradshteyn and I. Ryzhik, Table of integrals, series, and products, Academic Press, Inc., 1250 Sixth Avenue, San Diego CA 92101-4311, 5th ed., 1994.

[7] R. Haberman, Nonlinear transition layers - the second painlevé transcendent, Studies in Appl. Math., 57 (1977), pp. 247-270.

[8] - Energy bounds for the slow capture by a center in sustained resonance, SIAM J. Appl. Math., 43 (1983), pp. 244-256.

[9] R. Haberman AND E. Ho, Higher-order change of the hamiltonian (energy) for nearly homoclinic orbits, Methods and Applications of Analysis, 1 (1994), pp. 446-464.

[10] - Logarithmic correction to the probability of capture for dissipatively perturbed hamiltonian systems, Chaos, 5 (1995), pp. 374-384.

[11] J. Henrard, Dynamics Reported, vol. 2 (New Series), Springer-Verlag, Berlin Heidelberg, 1993, ch. The adiabatic invariant in classical mechanics, pp. 117-235.

[12] J. Kevorkian and J. Cole, Perturbation methods in applied mathematics, vol. 34 of Applied Mathematical Sciences, Springer-Verlag, 175 Fifth Avenue, New York, New York 10010, rev. and updated version ed., 1981.

[13] G. Kuzmak, Asymptotic solutions of nonlinear second order differential equations with variable coefficients, J. Appl. Math. Mech., 23 (1959), pp. 730-744.

[14] V. Melnikov, On the stability of the center for time periodic perturbations, Trans Moscow Math. Soc., 12 (1963), pp. 1-57.

[15] A. NEISHTADT, On the change in the adiabatic invariant on crossing a separatrix in systems with two degrees of freedom, J. Appl. Math. Mech., 51 (1987), pp. 586-592.

[16] D. Quinn, R. RAND, AND J. BRIDGe, The dynamics of resonant capture, Nonlinear Dynamics, 8 (1995), pp. 1-20.

[17] R. RAND, Symbolic computations and their impact on dynamics, vol. 205, Am. Soc. Mech. Eng., 1990.

[18] C. RoBinson, Sustained resonance for a nonlinear system with slowly varying coefficients, SIAM J. Math. Anal., 14 (1983), pp. 847-860.

[19] J. SANDERS AND F. VERHULst, Averaging methods in nonlinear dynamical systems, Applied Mathematical Sciences, Springer-Verlag, 175 Fifth Avenue, New York, New York 10010, 1985.

[20] J. Tennyson, J. Cary, and D. Escande, Change of the adiabatic invariant due to separatrix crossing, Phys. Rev. Lett., 56 (1986), pp. 2117-2120.

[21] A. Timofeev, On the constancy of an adiabatic invariant when the nature of the motion changes, Sov. Phys. JETP, 48 (1978), pp. 656-659. 